\title{
2D inverse modeling of residual gravity anomalies from Simple geometric shapes using Modular Feed-forward Neural Network
}

\author{
Ata Eshaghzadeh ${ }^{1,}$, Alireza Hajian ${ }^{2}$, \\ ${ }^{1}$ Graduate student of geophysics, Institute of Geophysics, University of Tehran, Iran \\ ${ }^{2}$ Department of Physics, Najafabad Branch, Islamic Azad University, Najafabad, Iran
}

\author{
Article history \\ Received September 14, 2017; accepted February 22, 2018. \\ Subject classification: \\ Modular Feed-forward Neural Network (MFNN); Module; Sigmoid function; Iron deposit; Gravity field; Iran.
}

\begin{abstract}
In this paper, we introduce a new method called Modular Feed-forward Neural Network (MFNN) to find the shape factor, depth and amplitude coefficient parameters related to simple geometric-shaped models such as sphere, horizontal cylinder, and vertical cylinder, which cause the gravity anomalies, in $2 D$ cross section. Using MFNN inversion results can determine the shape, depth and radius of a causative body. The design of MFNN consists of 3 similar one layer feed-forward neural networks (FNNs). Each feed-forward Neural Network which is as a module, first train using the back-propagation method for a parameter with synthetic gravity data and then to test the trained networks with new gravity data. The new approach has been tested first on synthetic data from different models using well-trained networks. The results of this approach show that the parameters values estimated by the modular inversion are almost identical to the true parameters. Furthermore, the noise analysis has been examined where the outputs of the inversion produce satisfactory results with $10 \%$ of random noise. The reliability of this approach is demonstrated for real gravity field anomalies measured over an iron deposit in Kerman province, Iran. MFNN inversion show the best shape for the underground mass is vertical cylinder with a depth of $21.18 \mathrm{~m}$ and a radius of $17.89 \mathrm{~m}$.
\end{abstract}

\section{Introduction}

Inversion of gravity data is nonunique in the sense that the observed gravity anomalies in the plane of observation can be explained by a variety of density distributions in different depths and various shapes. In the other words, different geometrical distributions of the subsurface mass can yield the same gravity field at the surface [Skeel 1947]. One way to solve this ambiguity is to assign a suitable geometry to the anomalous mass with a known density followed by inversion of gravity anomalies [Chakravarthi and Sundararajan 2004]. Al- though simple models may not be geologically realistic, they are usually are sufficient to analyze sources of many isolated anomalies [Abdelrahman and El-Araby 1993]. We can obtain a general view of the location of the structures by these simulations. The interpretation of such an anomaly aims essentially to estimate the parameters such as shape, depth, and radius of the gravity anomaly causative body such as geological structures , mineral mass and artificial underground structures. Several graphical and numerical methods have been developed for analyzing residual gravity anomalies caused by simple bodies, such as Saxov and Nygaard [1953] and Bowin et al. [1986]. The methods include, for example, Fourier transform [Odegard and Berg 1965, Sharma and Geldart 1968], Mellin transform [Mohan et al. 1986], Walsh transforms techniques [Shaw and Agarwal 1990], ratio techniques [Hammer 1977, Abdelrahman et al. 1989] and least-squares minimization approaches [Gupta 1983, Lines and Treitel 1984, Abdelrahman 1990, Abdelrahman et al. 1991]; effective quantitative interpretations using the least-squares method [Gupta 1983] based on the analytical expression of simple moving average residual gravity anomalies are yet to be developed. Abdelrahman and El-Araby [1993] introduced an interpretive technique based on fitting simple models convolved with the same moving average filter as applied to the measured gravity. A simple method proposed by Essa [2007] is used to determine the depth and shape factor of simple shapes from residual gravity anomalies along the profile. Another automatic method, the leastsquares method, was proposed by Asfahani and Tlas [2008], by which the depth and amplitude coefficient can be determined. 
Artificial Neural Networks (ANNs) are of major research interest at present, involving researchers of many different disciplines. Subjects contributing to this research include biology, computing, electronics, mathematics, medicine, physics, psychology and etc [Bichsel 2005]. The new method, the neural network, has been used in recent years for different branch of geophysics especially potential fields. For example, the location of buried steel drums is estimated from magnetic dipole source using supervised artificial neural network [Salem et al. 2001]. Depth and radius of subsurface cavities are determined from microgravity data using back propagation neural networks [Eslam et al. 2001]. Depth of the deposit was determined by applying a neural network [Hajian 2004]. A new approach in neural networks, Cellular Neural Network (CNN), has been proposed by Chua and Yang [1988], which is focused on 2D image processing. CNN was applied for separation of regional/residual potential sources in geophysics [Albora et al. 2001a, 2001b]. Forced Neural Networks for gravity anomaly was proposed by Osman et al. [2006, 2007]. Abedi et al. [2009] calculated the depth and radius of the simple geometry by the neural network from the gravity anomalies. Al-garni [2013] used MNN inversion for estimating the depth of the gravity anomaly source related to simple geometry separately. Eshaghzadeh and Kalantari [2015] have been proposed a new method based on feed-forward neural network for gravity field inverse modeling due to anticlinal structures.

In this paper, a new definition of the Modular Feed-forward Neural Network is proposed for inverse modeling of profile gravity data. We investigate the ability of the suggested approach in estimating the depth, shape and radius of buried sphere, infinite horizontal cylinder and semi-infinite vertical cylinder sources from calculated gravity data, with and without random noise. Then apply MFNN inversion for real gravity data set from Iran.

\section{Sources of simple geometry}

In gravity, fields of many simple bodies are symmetric about the location of the source. For example, the general gravity g effect caused by simple models (such as a sphere, an infinite horizontal cylinder, and a semi-infinite vertical cylinder as shown in Figure 1) is given as [Abdelrahman et al. 1989]:

$$
g\left(x_{i}, z, q\right)=K \frac{z^{m}}{\left(x_{i}^{2}+z^{2}\right)^{q}}
$$

Where $z$ is the depth, $m=1$ for a sphere or a horizontal cylinder and $\mathrm{m}=0$ for a vertical cylinder, $q$ is a

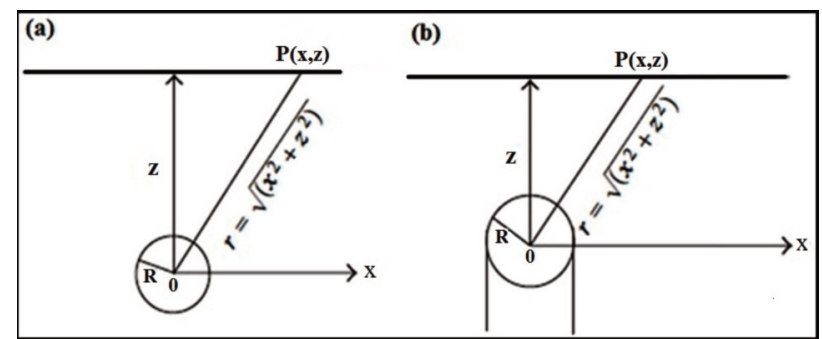

Figure 1. (a) sphere and horizontal cylinder models, (b) vertical cylinder model.

value (shape factor) characterizing the nature of the source ( $q=0.5$ for a vertical cylinder, $q=1$ for a horizontal cylinder, and $q=1.5$ for a sphere) and $K$ is an amplitude factor related to the radius $R$ and density contrast $\rho$ of the source, as:

$$
K=\left\{\begin{array}{l}
4 / 3 G \rho R^{3} \quad \text { for a sphere } \\
2 \pi G \rho R^{2} \quad \text { for a horizontal cylinder } \\
\pi G \rho R^{2} \quad \text { for a vertical cylinder }
\end{array}\right.
$$

\section{Modular Feed-forward Neural Network}

Generally, a Neural Network is taught by a training set of a group of examples as it learns to estimate one or more parameters described by the example patterns. Neural networks can be classified in supervised (associative) and unsupervised (self-organization). The main difference is that in the case of the supervised neural networks the learning algorithm uses input-output training data to model the dynamic system, on the other hand, in the case of unsupervised neural networks only the input data is given. In the case of an unsupervised network, the input data is used to make representative clusters of all the data.

A typical NN consists of a minimum of three layered nonlinear problems: an input layer, a hidden layer and an output layer (Figure 2).

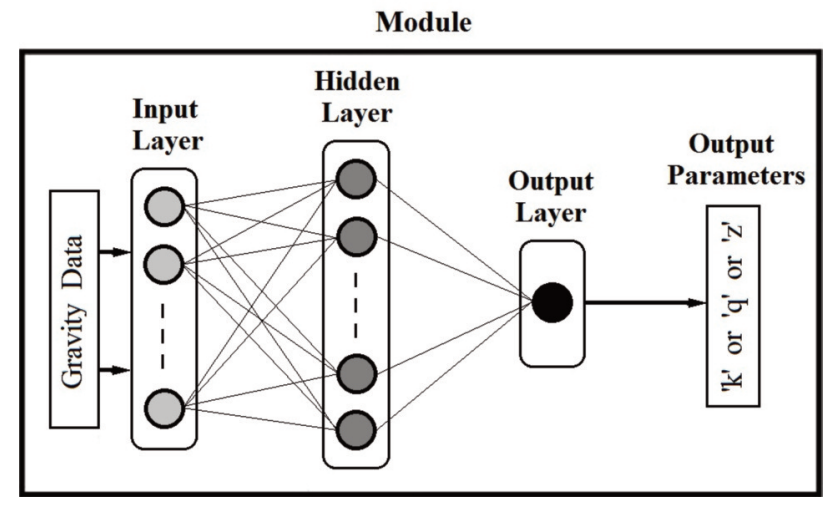

Figure 2. Architecture of feed-forward neural network (Module). 
In this paper, we have proposed a new structure of the Modular Neural Network that consists of some monolayer feed-forward neural networks (FNN), each one trained with a supervised method with distinct training data set. Each feed-forward Neural Network has the same structure and is trained under the same conditions. In other words, a Modular Neural Network has been formed with some parallel feed-forward neural networks as each independent FNN serves as a module (local expert) operates on separate inputs to accomplish some subtask of the task that the network wishes to implement (Figure 3). Response integration is required to combine the outputs of all the modules in the modular network.

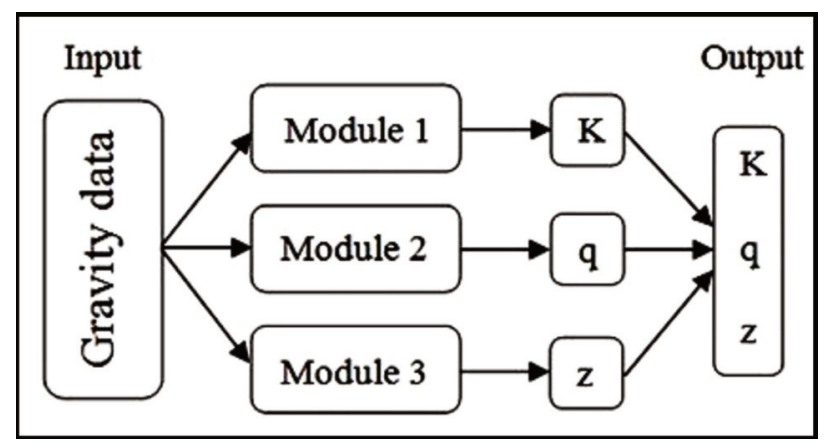

Figure 3. Architecture of a modular feed-forward neural network (MFNN) with three module.

In a feed-forward network, the connections between neurons are in one direction. Each neuron consists of a vector of modifiable weights or connection strengths. The task of a neuron is to map a given input vector into a single output that is transmitted to other neurons. A feed-forward network is usually arranged in the form of layers. In such a layered feed-forward network, there is no connection between the neurons in the same layer, and there is no feedback between layers. The neuron uses an activation function to transform the net input into a single output. An activation function commonly used in an FNN is the sigmoid function. This function has a linear transition part that exponentially reaches one of two states. The sigmoid function is given by

$$
f(x)=\left(1+e^{-x}\right)^{-1}
$$

In the ANN context, the process of finding optimum weights is described as network training and the set of input-output patterns is the training set. The purpose of learning a mapping between known input-output patterns for a given problem is to apply the trained network to input patterns with unknown outputs later on. From this viewpoint, an FNN can be described as a non-linear function; the training process is an optimization problem where network weights are the parameters to be estimated [Macias et al. 2000].

The most widely used training method is known as the back-propagation method. The simplest implementation of back-propagation learning updates the network weights and biases in the direction in which the performance function decreases most rapidly. The backpropagation method produces a least-squares fit between the actual network output and a desired output by computing a local gradient in terms of the network weights [Rummelhart et al. 1986]. During the training process, the measure of the error is given by [Macias et al. 2000]

$$
E=\frac{1}{2} \sum_{q=1}^{O} \sum_{k=1}^{M}\left(y_{k}^{q}-o_{k}^{q}\right)^{2}
$$

Where $o_{k}^{q} s$ the $k$ th output of the network and $y_{k}^{q}$ is the $k$ th value which is expected, $k=1, \ldots, \mathrm{M}$. After learning, a neural network represents a complex relationship, and possesses the ability for generalization.

\section{MFNN training}

In a neural network, we can train the network by using input and output data (training vector). Here, the inputs of feed-forward neural networks are gravity data of synthetic profile that are generated by forward modeling. Outputs are the geometric parameters of a model.

The new approach will integrate the information from three main modules, one for each of the three geometric parameters (Figure 3). Therefore each module in MFNN architecture estimate one of the shape factor, depth or amplitude coefficient parameters from the gravity data. The final decision is based on the results of the three modules. This strategy is caused instead of training the entire dataset at once, data divide into smaller subsets as can define some smaller FNN, thus increase the learning speed and improve the output accuracy.

The number of training samples should be sufficient to reach the desired training accuracy. To generate training samples, forward modeling is performed at 33 points over $66 \mathrm{~ms}$ profile with two-ms interval. We have considered the ranges from 8.0 to $25 \mathrm{~ms}$, with 10 points for the depth $(z)$ parameter, the ranges from 0.4 to 1.6 with 10 points for shape factor $(q)$ parameter and the ranges from 10 to 210 units, with 22 points for the amplitude coefficient $(k)$ parameter. The parameters are selected in aforementioned ranges disorderly. The choice the expected ranges of the parameters is based on the behavior of measured field data and the geological information from the area under consideration.

Because each module is taught for estimating one 


\begin{tabular}{|c|c|c|c|c|c|c|c|c|c|}
\hline \multirow{2}{*}{$\begin{array}{l}\text { Model } \\
\text { Parameter }\end{array}$} & \multicolumn{3}{|c|}{ Sphere } & \multicolumn{3}{|c|}{ horizontal cylinder } & \multicolumn{3}{|c|}{ vertical cylinder } \\
\hline & Initial & $\begin{array}{l}\text { Without } \\
\text { noise }\end{array}$ & $\begin{array}{l}\text { with } \\
\text { noise }\end{array}$ & Initial & $\begin{array}{l}\text { Without } \\
\text { noise }\end{array}$ & $\begin{array}{l}\text { with } \\
\text { noise }\end{array}$ & Initial & $\begin{array}{l}\text { Without } \\
\text { noise }\end{array}$ & $\begin{array}{l}\text { with } \\
\text { noise }\end{array}$ \\
\hline Depth (z) & 20 & 20.07 & 22.72 & 22 & 21.87 & 23.08 & 15 & 15.062 & 15.64 \\
\hline Shape factor $(q)$ & 1.5 & 1.495 & 1.453 & 1 & 1 & 0.986 & 0.5 & 0.503 & 0.467 \\
\hline $\begin{array}{l}\text { Amplitude } \\
\text { coefficient }(k)\end{array}$ & 110 & 109.89 & 112.32 & 25 & 24.83 & 26.87 & 12 & 12.03 & 10.86 \\
\hline
\end{tabular}

Table 1. Inverted parameters from MFNN for synthetic gravity data. The depth unit is $\mathrm{m}$ and the amplitude coefficient unit for sphere model is $\mathrm{mGal} . \mathrm{m}^{2}$ and for horizontal and vertical cylinder is $\mathrm{mGal} . \mathrm{m}$.

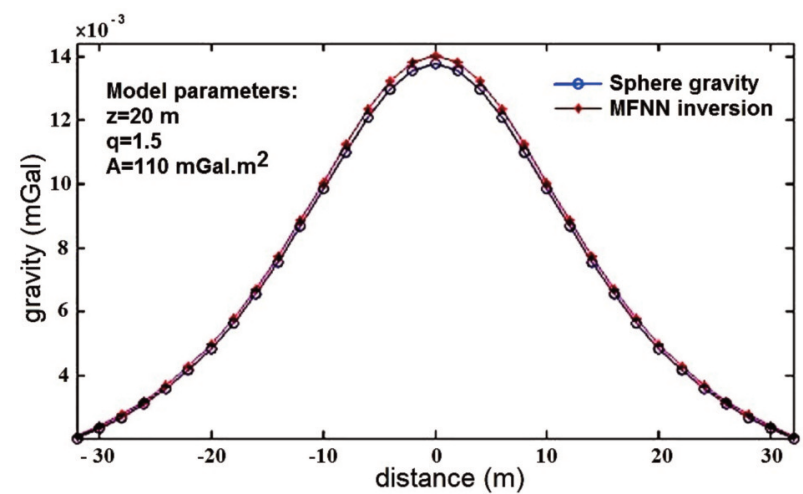

Figure 4. The gravity response of the sphere model (blue curve) and MFNN inversion (red curve).

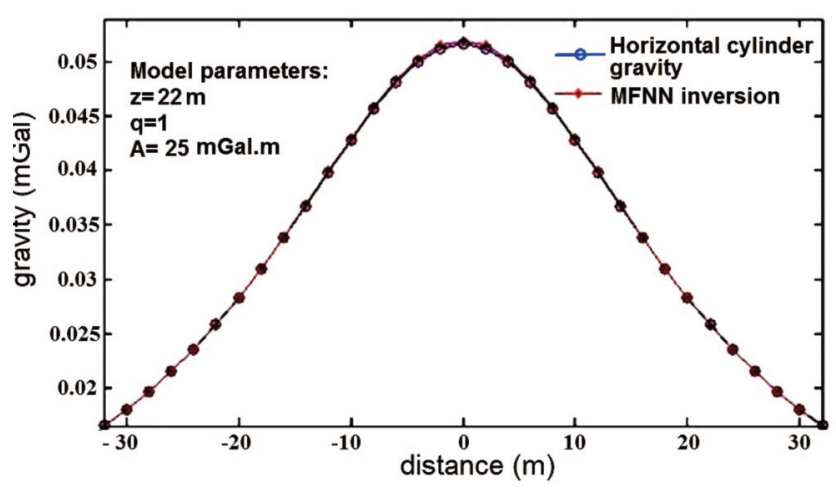

Figure 5. The gravity response of the horizontal culinder model (blue curve) and MFNN inversion (red curve).

of the parameters, in the forward modeling phase for generating training data, to avoid repeating a constant value for a parameter in training vectors, we have used from the rand command in Matlab. Two thousand two hundred training patterns are calculated. Each training vector contains thirty four samples.

The selected modules are a $(33,20,1) \mathrm{FNN}$. It means a feed-forward neural network with 33 neurons

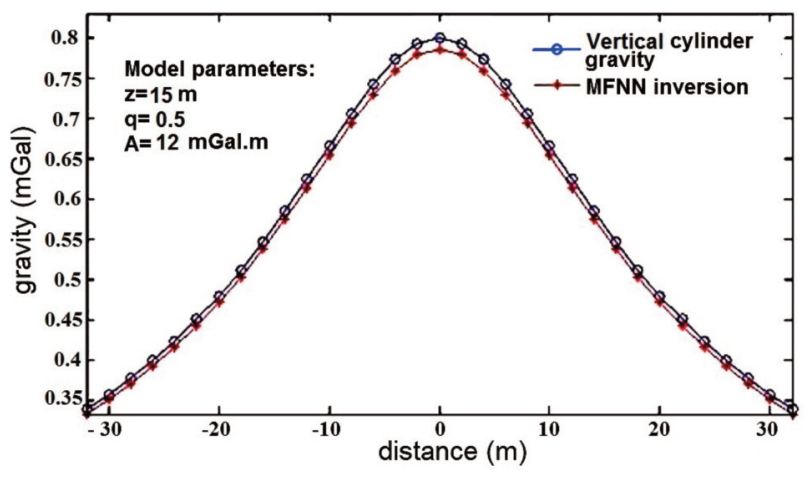

Figure 6. The gravity response of the vertical culinder model (blue curve) and MFNN inversion (red curve).

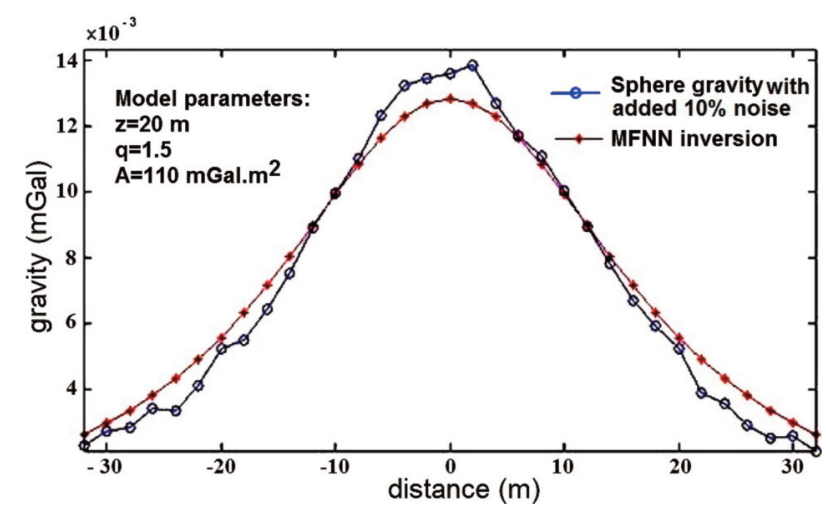

Figure 7. The gravity response of the sphere model which corrupted with $10 \%$ random noise (blue curve) and MFNN inversion (red curve).

as inputs (gravity data), 20 neurons in the hidden layer and 1 neuron as output layer, because outputs are one of the $q, K$ or $z$ parameters. The error convergence during network training depends on the number of neurons in the hidden layer of the network. More important than the error reduction is the ability of the network to predict answers successfully from new data which depends on both the number of neurons defining 


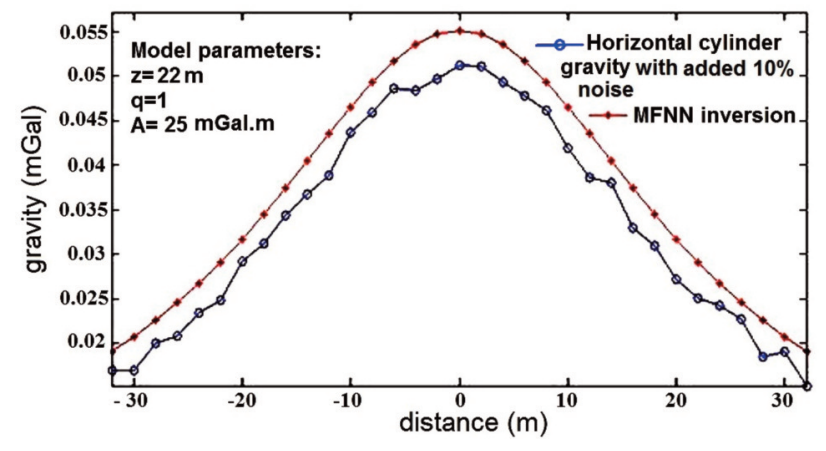

Figure 8. The gravity response of the horizontal culinder model which corrupted with $10 \%$ random noise (blue curve) and MFNN inversion (red curve).

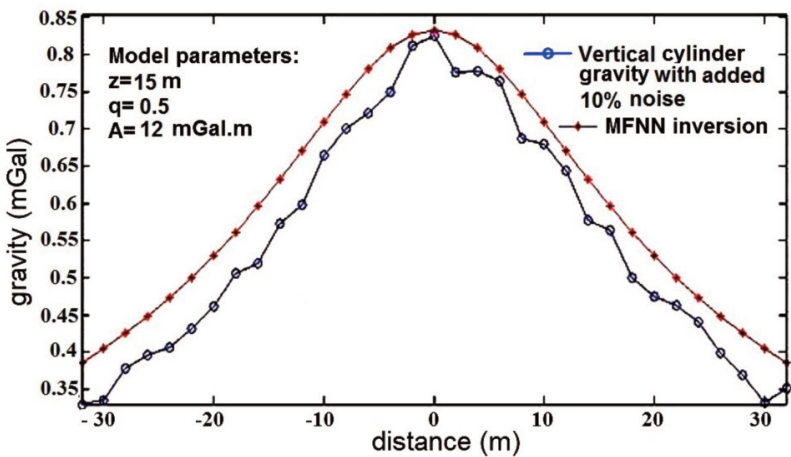

Figure 9. The gravity response of the vertical culinder model which corrupted with $10 \%$ random noise (blue curve) and MFNN inversion (red curve).

the network and the number of examples used for training [Macias et al. 2000]. The number of hidden neurons can be defined more exactly by trial-and-error methods in the calculated limits.

\section{Synthetic models}

After correct training of the modules, by entering residual gravity anomaly profile of a synthetic model or real gravity profile into trained MFNN, geometric parameters of the model are obtained. In this study, we have trained and saved three FNN as modules for inverse modeling of the sphere, infinite horizontal cylinder and semi-infinite vertical cylinder shapes.

Those have assumed that the sphere model to have parameters $\mathrm{z}=20 \mathrm{~m}, \mathrm{q}=1.5$ and $\mathrm{K}=110 \mathrm{mGal} . \mathrm{m}^{2}$, horizontal cylinder model to have parameters $\mathrm{z}=22 \mathrm{~m}, \mathrm{q}=1$ and $\mathrm{K}=25 \mathrm{mGal} . \mathrm{m}$ and vertical cylinder model to have parameters $\mathrm{z}=15 \mathrm{~m}, \mathrm{q}=0.5$ and $\mathrm{K}=12 \mathrm{mGal} . \mathrm{m}$. The gravity effects of the sphere, horizontal cylinder and vertical cylinder models and generated gravity responses from MFNN inversion are shown in Figures 4, 5 and 6, respectively. The Table 1 shows the estimated parameters from the modules output.

The trained networks ability was also tested using corrupted synthetic gravity data with $10 \%$ random noise.
The gravity data profile of the sphere, horizontal cylinder and vertical cylinder models with added noise and Prediction results from MFNN inversion responses are shown in Figures 7, 8 and 9, respectively. The inverted parameters from corrupted data with noise are tabulated in Table 1. The satisfactory results have been obtained.

\section{Field example}

The area under study is located in the north of Kerman province, Iran, as encompass an area about $270 \mathrm{~m}$ by $130 \mathrm{~m}$ (Figure 10). Kerman province can be a part of Central Iran zone in structural units and extent of sedimentary basins viewpoint. Paleozoic to Mesozoic geological formation of the study region consists of dolomite and dolomitic limestone, orbitolina limestone with marl, alternations of marly biomicrite with marl, sandy micrite and siltstone. Quaternary sediments include sand dunes and sheets, silt and clay. Percambrian outcrops comprise volcanic rock, quartzite, sandstone and shale.

Figure 11 shows the geological map of region under investigation as the exploration area lie in the district confined by the black rectangle. The main iron ores

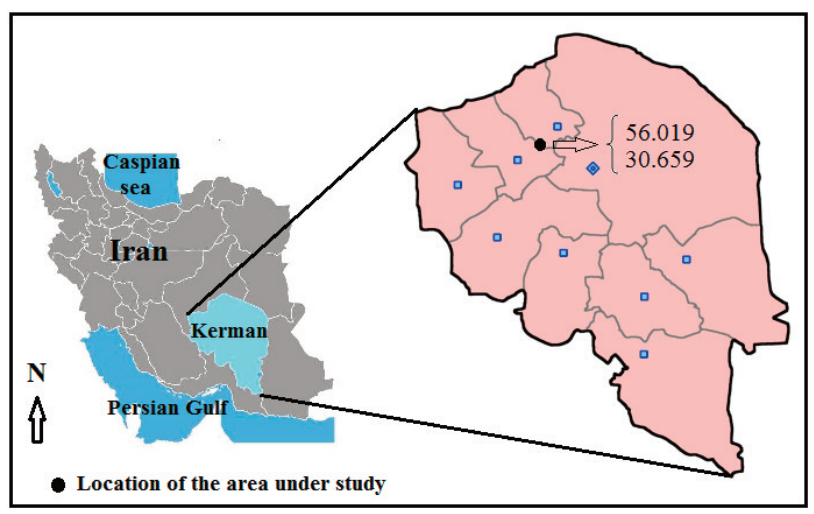

Figure 10. The location of the area under study.

in this area are from the oxide group consisting of hematite and magnetite. The aim of the gravity field measurments is underground metal mass detection.

The gravity reading was done with a station separation of about $5 \mathrm{~m}$, along 13 north-south profiles with a distance of about $20 \mathrm{~m}$. After gravity corrections, the complete Bouguer gravity anomalies map will be obtained (Figure 12). The Bouguer gravity field demonstrate a increasing trend from south-east to north-west.

As we are looking for the local gravity anomalies, residual gravity anomalies are computed removing a trend (degree 2) from the Bouguer anomalies. Figure 13 shows the residual gravity anomalies map of the area under investigation. Due to presence the non-metallic minerals and other elements in the iron deposite, the density of the iron ore deposite is about $4.5 \mathrm{gr} / \mathrm{cm}^{3}$, 
Q : Low terrace, young gravel fans

Q1: High terrace, old gravel fans

$\mathrm{Ca}$ : Dolomite, dolomite limestone

$\mathrm{Cd}:$ Limestone, dolomite

$\mathrm{C}_{\mathrm{m}}$ : Mainly dolomite and limstone, shale

$\mathrm{Dh}$ : Limestone, marly limestone

$D_{p}$ : Quartz sandstone, dolomite, shale

$\mathrm{K} 1$ : Marly biomicrite, siltstone

$\mathrm{K}_{2}$ : Limstone, sandy limstone, marly limestone

$\mathrm{K} 3$ : Orbitolina limestone

$\mathrm{P}_{\mathrm{c} 2}$ : Mainly volcanic rock, quartzite, shale

$\mathrm{P}_{\mathrm{j}}$ : Limstone, locally marmorized bedded dolomite

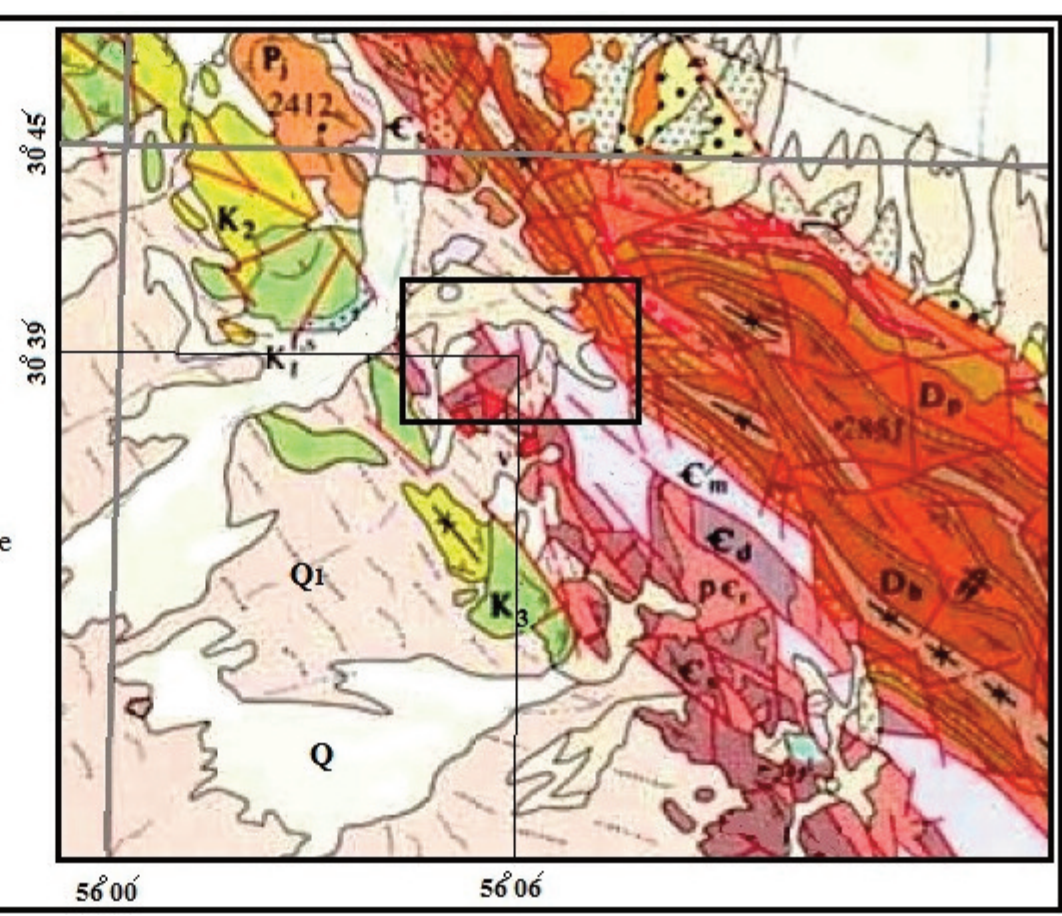

Figure 11. The geological map of the region under investigation.

thus the iron deposite mass has a density contrast about $1.9 \mathrm{gr} / \mathrm{cm} 3$ than surrounding sediments with a density of about $2.6 \mathrm{gr} / \mathrm{cm} 3$. The positive anomalies over the residual gravity anomalies map of the area under evaluation indicate the causative masses with positive density contrast such as metal minerals.

Profile A with direction of NE-SW in residual anomaly map is considered for inverse modeling with MFNN algorithm (Figure 13). The gravity sampling was done at 33 points over profile $\mathrm{A}$ with a distance of $2 \mathrm{~m}$.

The gravity field variations along the profile $\mathrm{A}$ and the gravity response related to the estimated parameters by MFNN inversion are shown in Figure 14. The neural network inversion shows that the estimated depth is $21.18 \mathrm{~m}$, shape factor is 0.587 and the amplitude coefficient is $12.74 \mathrm{mGal} . \mathrm{m}$. thus can simulate the anomaly source shape geometrically to vertical cylinder. The standard error (SE) is used as a statistical preference criterion in order to compare the observed and calculated values [Asfahani and Tlas 2008],

$$
S E=\sqrt{\frac{\sum_{i=1}^{N}\left(g_{i}^{o}-g_{i}^{c}\right)^{2}}{N}}
$$

where $g_{i}^{o}$ and $g_{i}^{c}(i=1, \ldots, N)$ are the observed and the calculated values at the points $x_{i}(i=1, \ldots, N)$, respectively.

The standard error $(S E)$ between the real gravity data and evaluated gravity is 0.0711 . For computing the amplitude coefficient of a vertical cylinder, with attention to relation 2, we can estimate the approximate ra- dius of the anomaly causative mass, as

$$
\begin{aligned}
& \pi G \rho R^{2}=12.74 \times 10^{-5} \mathrm{~m}^{2} / \mathrm{s}^{2} \\
& 3.98 \times 10^{-7} R^{2}=12.74 \times 10^{-5} \\
& R=17.89 \mathrm{~m}
\end{aligned}
$$

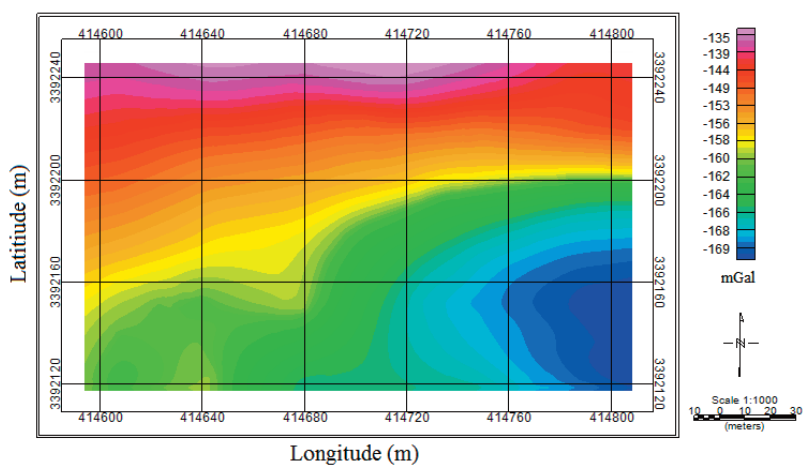

Figure 12. The Bouguer gravity anomaly map of the area under study.

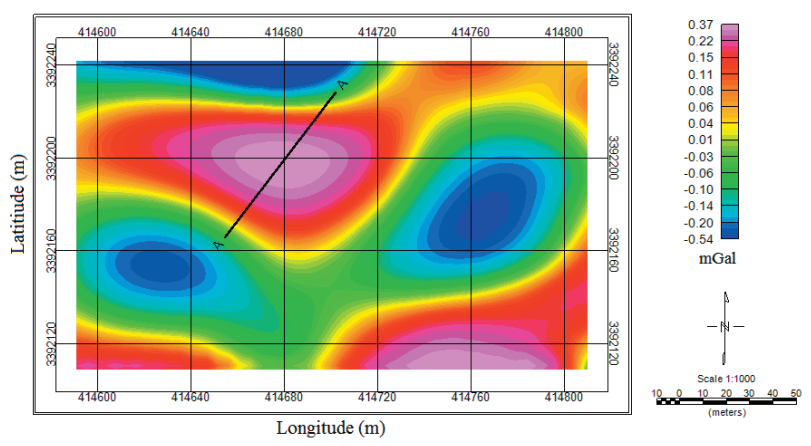

Figure 13. The residual gravity anomalies map of the area under study. 
The resulted parameters from inverting the real gravity data profile using MFNN have been shown in Table 2.

\begin{tabular}{lc}
\hline Parameter & Estimated values \\
\hline Depth (m) & 21.18 \\
$\begin{array}{l}\text { Amplitude coefficient } \\
\text { (mGal.m) }\end{array}$ & 12.74 \\
Shape factor & 0.587 \\
Radius (m) & 17.89 \\
\hline
\end{tabular}

Table 2. Estimated parameters from the inverse modeling of the real gravity data profile by MFNN. anomaly causative body actually has not a complete vertical cylinder shape.

\section{Discussion and conclusions}

In this paper, a new definition of Modularity for neural network architecture has been proposed. The modular neural network is designed to separate the functions that cause interference problems by having independent modules assigned to each function. Modules are groups of unconnected neurons, each connected to the same set of nodes. The concept of modularity is linked to the notion of local computation, in the sense that each module is an independent system and interacts with others in a whole architecture, in order to perform a given task.

Modules can be trained in parallel which reduces

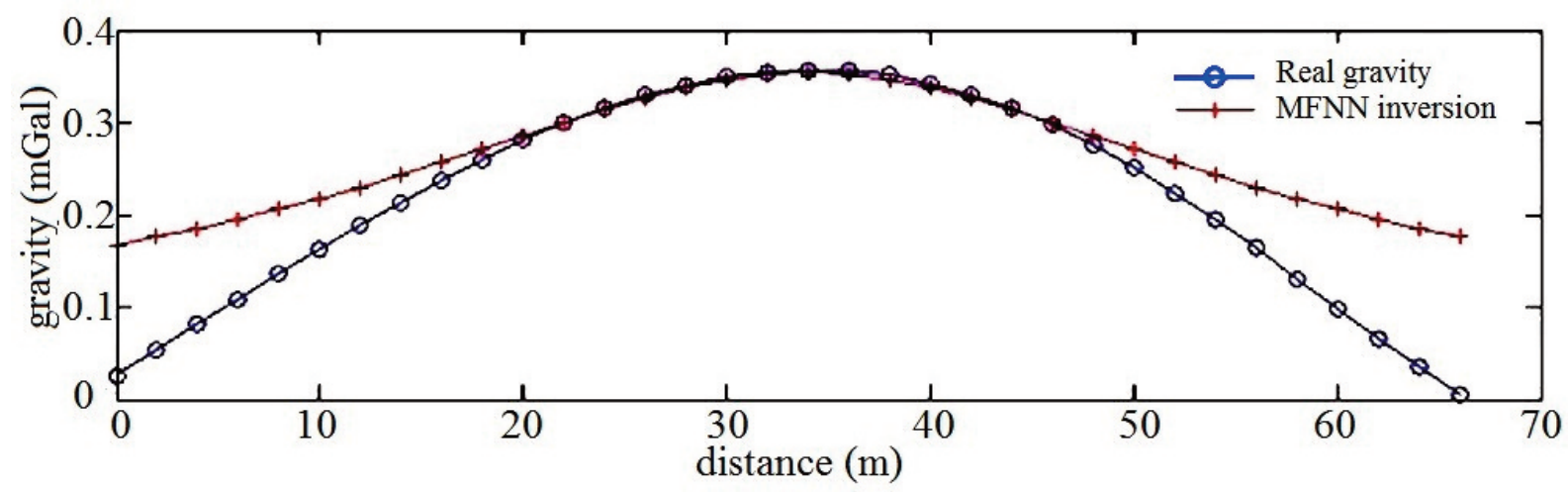

Figure 14. Observed anomaly of the profile A cross-section (blue curve) and calculated gravity from the estimated parameters of MFNN inversion (red curve).

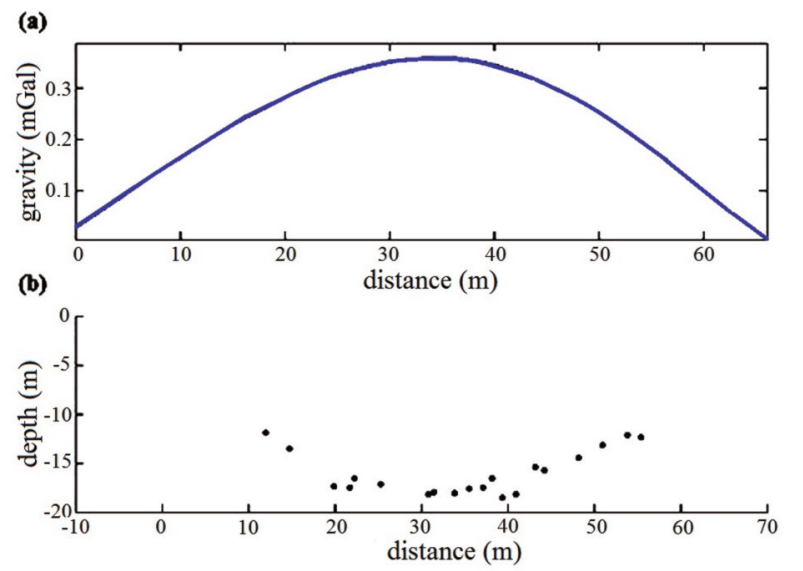

Figure 15. (a) Observed anomaly of the profile A cross-section, (b) the depth estimates using the Euler method.

Figure 15(b) show the depth estimates from Euler deconvolution method using a structural index of 1 and a window size of 3 points for observed anomaly of the profile A cross-section (Figure 15(a)). The maximum depth is about $19 \mathrm{~m}$. In comparison with the evaluated depth from MFNN inversion, a difference of about $2 \mathrm{~m}$ is seen. This difference is because of the underground training times substantially and additional modules can be added without the need to retrain the others. During training the weights and biases of the network are iteratively adjusted to minimize the network performance function. The default performance function for feed-forward networks is mean square error.

The main advantage of using MFNN for gravity field interpretation is that once MFNN has been trained for shape factor, depth and amplitude coefficient parameters of the simple models such as a sphere, an infinite horizontal cylinder, and a semi-infinite vertical cylinder, it has the ability to interpret gravity field measurements very rapidly. In this approach, MFNN do not need to train for each simple shape separately.

The modular feed-forward neural network was investigated for inverse modeling the gravity response of the buried body with simple geometry. The efficiency of MFNN was evaluated with adding $10 \%$ random noise to the synthetic gravity data. The results of the modular neural network approach illustrate MFNN excellent performance. We employ MFNN for the real gravity data from Iran. The inverted parameters from 
the real gravity data profile show the best form for underground deposit mass is the vertical cylinder shape, as is located at a depth of $21.18 \mathrm{~m}$. We have compared MFNN with the Euler method. The Euler solutions show a depth of about $19 \mathrm{~m}$ for the anomaly source with assuming a structural index of 1 . Based on geological surveys done in the region under study, these depths are expected. The successful application of MFNN inversion to the synthetic and gravity field data demonstrated the reliability and the validity of this approach.

\section{References}

Abdelrahman, E. M., Bayoumi, A. I., Abdelhady, Y. E., Gobashy, M. M., and El-Araby, H. M., 1989. Gravity interpretation using correlation factors between successive least-squares residual anomalies. Geophysics, 54, 1614-1621.

Abdelrahman, E. M., 1990. Discussion on "A leastsquares approach to depth determination from gravity data” by O. P. Gupta”. Geophysics, 55, 376-378.

Abdelrahman, E. M., Bayoumi, A. I., and El-Araby, H. M., 1991. A least-squares minimization approach to invert gravity data. Geophysics, 56, 115-1 18 .

Abdelrahman, E. M., and El-Araby, T. M., 1993. A leastsquares minimization approach to depth determination from moving average residual gravity anomalies. Geophysics, 58(12), 1779-1784.

Abdelrahman, E. M., and El-Araby, H. M., 1993. Shape and depth solutions from gravity using correlation factors between successive least-squares residuals. Geophysics, 59, 1785-1791.

Abedi, M., Afshar, A., Ardestani, V. E., Norouzi, G. H., and Lucas, C., 2009. Application of Various Methods for 2D Inverse Modeling of Residual Gravity Anomalies. Acta Geophysica, 58(2), 317-336.

Albora, A.M., Ucan, O.N., Özmen, A., and Ozkan, T., 2001a. Separation of Bouquer anomaly map using cellular neural network", J. Appl. Geophys., 46, 129142.

Albora, A.M., Özmen, A., Ucan, O.N., 2001b. Residual separation of magnetic fields using a cellular neural network approach. Pure Appl. Geophys., 158, 17971818.

Al-Garni, M.A., 2013. Inversion of residual gravity anomalies using neural network. Arab J Geosci, 6,1509-1516.

Asfahani, J., and Tlas, M., 2008. An automatic method of direct interpretation of residual gravity anomaly profiles due to spheres and cylinders," Pure and Applied Geophysics, 165 (5), 981-994.
Bichsel, M., 2005. Image processing with optimum neural networks", IEEE International Conference on $\mathrm{Ar}$ tificial Neural Networks, 513, 374-377.

Bowin, C., Scheer, E., and Smith, W., 1986. Depth estimates from ratios of gravity, geoid, and gravity gradient anomalies. Geophysics, 51, 123-136.

Chakravarthi, V., and Sundararajan, N., 2004. Ridge-regression algorithm for gravity inversion of fault structures with variable density. Geophysics, 69(6), 1394-1404.

Chua, L.O., and Yang, L., 1988. Cellular neural networks: Theory", IEEE Trans. Circuits Syst., 35(10), 1257-1272.

Eshaghzadeh, A., and Kalantari, R.A., 2015. Anticlinal Structure Modeling with Feed Forward Neural Networks for Residual Gravity Anomaly Profile. 8th congress of the Balkan Geophysical Society, DOI: 10.3997/2214-4609.201414210.

Eslam, E., Salem, A., and Ushijima, K., 2001. Detection of cavities and tunnels from gravity data using a neural network. Explor. Geophys. 32, 204-208.

Essa, K. S., 2007. A simple formula for shape and depth determination from residual gravity anomalies. Acta Geophysica, 55(2),182-190.

Gupta, O. P., 1983. A least-squares approach to depth determination from gravity data. Geophysics, 48, 357360.

Hajian, A.R., 2004. Depth estimation of gravity data by neural network", M. Sc. thesis, Tehran University, Iran (Persion).

Hammer, S., 1977. Graticule spacing versus depth discrimination in gravity interpretation. Geophysics, 42, 60-65.

Lines, L. R., and Treitel, S., 1984. A review of leastsquares inversion and its application to geophysical problems. Geophys. Prosp., 32, 159-186.

Macias, C., Sen, M. K., and Stoffa, P. L., 2000. Artificial neural networks for parameter estimation in geophysics. Geophys Prospect., 48,21-47.

Mohan, N. L., Anandababu, L., and Rao, S., 1986. Gravity interpretation using the Melin transform. Geophysics, 51, 114-122.

Odegard, M. E., and Berg, J.W., 1965. Gravity interpretation using the Fourier integral. Geophysics, 30, 424-438.

Osman, O., Albora A.M., and Ucan, O.N., 2006. A new approach for residual gravity anomaly profile interpretations: Forced Neural Network (FNN). Ann. Geofis., 49,6 .

Osman, O., Albora A.M., and Ucan, O.N., 2007. Forward modeling with Forced Neural Networks for gravity anomaly profile”, Math. Geol., 39, 593-605. 
Rummelhart, D.E., Hinton, G.E., and Williams, R.J., 1986. Learning internal representation by backpropagating errors. Nature, 332, 533-536.

Salem, A., Ravat, D., Johnson, R., and Ushijima, K., 2001. Detection of buried steel drums from magnetic anomaly data using a supervised neural network. J. Environ. Eng. Geophys., 6, 115-122.

Saxov, S., and Nygaard, K. 1953. Residual anomalies and depth estimation. Geophysics, 18, 913-928.

Sharma, B., and Geldart, L. P., 1968. Analysis of gravity anomalies of two-dimensional faults using Fourier transforms. Geophys. Prosp., 77-93.

Shaw, R. K., and Agarwal, N. P., 1990. The application of Walsh transforms to interpret gravity anomalies due to some simple geometrically shaped causative sources: A feasibility study. Geophysics, 55, 843-850.

Skeels, D. C., 1947. Ambiguity in gravity interpretation. Geophysics, 12, 43-56.

*Corresponding author: Ata Eshaghzadeh,

Graduate student of geophysics, Institute of Geophysics,

University of Tehran, Iran;

email: eshagh@alumni.ut.ac.ir.

(C) 2018 by the Istituto Nazionale di Geofisica e Vulcanologia. All

rights reserved. 